\title{
La confusion des genres : album « mixte » et littératie
}

\author{
Patricia Richard-Principalli et Marie-Françoise Fradet \\ Université Paris Est, EA 4384 Circeft, Université Paris 8, UPEC, 94010 Créteil Cedex, France
}

\begin{abstract}
Résumé. Dans le cadre des travaux de Circeft-Escol sur la littératie scolaire et en particulier sur les supports d'apprentissage actuels, cette analyse envisage l'étude d'un texte « composite » dans une classe de Cours préparatoire de l'école élémentaire en ZEP, en juin 2012. Une si petite graine, d'Eric Carle (2003, Mijade Jeunesse), donne ainsi à lire l'évolution d'une graine, de la germination à la fleur, présentée dans sa nature cyclique liée à la succession des saisons. Sa visée semble donc informative. Cependant, cette visée est contaminée par une pratique narrativisée de l'information qui inscrit aussi l'album dans une démarche esthétique. Nous analysons précisément l'album dans sa tension entre informatif et narratif, en le définissant comme album « mixte », caractérisé par sa visée double, qui se traduit par une structure et une énonciation doubles. La mise en œuvre de l'enseignant dans la classe insiste sur l'aspect informatif. Or les réponses produites par les élèves montrent que ceux qui ont le mieux acquis les connaissances liées au monde du vivant sont ceux qui ont le mieux compris la complexité de l'album et sa partie fictionnelle, soit les élèves appartenant au groupe de niveau des meilleurs.
\end{abstract}

Dans le cadre des travaux de l'équipe $\mathrm{ESCOL}^{1}$ sur la littératie scolaire et notamment sur les supports actuels, cette étude poursuit l'étude du composite, caractérisé par le pluricodage et la discontinuité de l'information [1] à l'œuvre dans un album de jeunesse représentatif selon nous d'un type de composite particulier et fréquent dans la production jeunesse actuelle ${ }^{2}$, qui associe une visée narrative à une visée documentaire. L'album que nous analysons ici cherche par le biais d'une histoire à faire découvrir à de jeunes lecteurs des connaissances sur le monde du vivant.

Il se trouve donc à la croisée de l'histoire d'un individu relevant du singulier propre à la fiction [2], et de l'histoire de la perpétuation d'une espèce, qui caractérise l'approche biologique [3], relevant du générique propre au texte informatif [4]. L'album constitue ainsi un objet hétérogène qu'il n'est pas facile de caractériser, a fortiori pour un jeune lecteur.

Cela étant, nous nous demanderons en quoi ce type d'album, que nous qualifierons de « mixte », permet à des élèves de construire des connaissances sur le monde, en faisant l'hypothèse que cet apprentissage n'est possible pour tous les élèves que si sa spécificité est comprise et si la fiction est prise en compte. Nous nous appuierons sur l'analyse d'un travail observé dans une classe de CP (cours préparatoire, première année de l'école élémentaire), en ZEP, en juin 2012, s'appuyant sur l'album intitulé Une si petite graine (Eric Carle, Mijade Jeunesse, 2003).

\footnotetext{
${ }^{1}$ http://www. circeft.org/?-accueil-escol-

2 Voir la collection « Archimède », L'École des loisirs.
}

This is an Open Access article distributed under the terms of the Creative Commons Attribution License 4.0, which permits unrestricted use, distribution, and reproduction in any medium, provided the original work is properly cited. 


\section{Une si petite graine}

Une si petite graine donne à lire l'évolution d'une graine, de la germination à la fleur, présentée dans sa nature cyclique liée à la succession des saisons. Sa visée semble donc informative. Cependant, cette visée est contaminée par une pratique narrativisée de l'information qui la vectorise. Le récit ici n'est possible que parce qu'il y a une transformation de fait.

La graine, sans être anthropomorphisée, est dotée des caractéristiques d'un personnage, perceptibles dès le titre, Une si petite graine. Le superlatif absolu souligne ici la taille de la graine, et constitue une caractéristique stylisante, qui induit qu'il va s'agir d'une histoire, où le processus de transformation lié au récit redouble le phénomène scientifique de croissance.

Cette inférence est explicitée par l'illustration qui représente une fleur (et non pas une graine) et par la mise en page qui met le titre au cœur de la fleur, focalisant sur le lien entre la graine et la fleur et sur le rôle essentiel de la petite graine.

Ce statut se confirme dès l'incipit. Il se renforce tout au long de l'histoire qui se déroule selon cinq étapes qui sont aussi les cinq étapes du cycle de la croissance, dont la correspondance nécessite une inférence puisqu'elles ne sont pas explicitement mises en lien. En effet, si les graines sont éliminées les unes après les autres par les aléas de la vie naturelle, c'est la petite graine qui, illustrant ce que tout documentaire retiendrait pour la croissance, porte la fiction, à travers un destin de croissance réussie.

\subsection{L’automne : voyage/dissémination}

Le début de l'album est marqué par la mise en place d'une énonciation double : un discours fictionnel qui intronise le personnage et un discours informatif porté par la question finale de la première page, à l'adresse du lecteur : « où vont-elles toutes ces graines ? »

L'alternance du singulier et du collectif, du défini et de l'indéfini (« la toute petite graine »vs. « une graine » ou « les graines ») marque la double énonciation qui rapporte d'une part les dangers auxquels échappe « la toute petite graine » et d'autre part les aléas de la dissémination que subissent les autres graines : les rayons du soleil, la glace des sommets, l'océan, le désert, la voracité des oiseaux.

\subsection{L'hiver : repos/dormance}

Une seule double page est consacrée à cette étape, pourtant aussi essentielle que les autres, sous le repos apparent : pendant l'hiver, les graines se reposent dans le sol couvert de neige. L'opposition se poursuit entre le sort des autres graines, soumises à la voracité d'une souris, et celui de « la toute petite graine », « qui dort tranquillement », échappant une fois de plus au danger.

\subsection{Le printemps : métamorphose/transformation ${ }^{3}$}

Au moment où les graines, traitées comme des éléments naturels, se transforment en plantes, « la petite plante », vue, elle, comme un personnage, devient acteur de sa transformation comme le montre la subjectivité qui lui est prêtée (« elle se décide »).

C'est là que s'observe le mieux le phénomène de dédoublement : les éléments naturels suivent les lois de la nature, tandis que la petite graine, qui semble toujours « en décalage », est dotée d'une vie personnelle. Malgré la brièveté du texte qui lui est consacré à chaque nouvelle aventure et malgré son apparente faiblesse (elle est à peine visible dans l'illustration), c'est elle en effet qui porte le récit et son intérêt, illustrant un scénario de gagnant, propre aux contes qui reposent sur le stéréotype (Dufays, 1994) du triomphe du faible sur le fort, tel qu'il court depuis les textes bibliques jusqu'à nos jours.

\footnotetext{
${ }^{3}$ Nous prenons ici les deux termes dans un emplois pécifique : « métamorphose » pour renvoyer au monde du conte, « transformation » pour renvoyer au phénomène naturel de la croissance.
} 
La fiction se manifeste plus puissamment encore à partir du moment où l'homme entre en scène dans l'histoire (p. 11). L'arrivée de l'homme dans l'univers de la petite plante se fait de façon graduée (pied, main, couple) jusqu'à ce qu'elle se retrouve au centre de l'univers magique propre au conte.

\subsection{L'été : apothéose/floraison}

Dès lors, le soleil et la pluie ne sont plus des opposants mais des adjuvants (p. 13).

Dominant « les arbres, les gens et les maisons », la «si petite graine », à l'instar du haricot magique, devient une «fleur géante », reconnue et admirée de tous. C'est une véritable apothéose, comparable à celle du soleil dans l'illustration, qui donne tout son sens à la première de couverture.

\subsection{L'automne : mort et nouvelles naissances/reproduction}

Avec le retour de l'automne, la plante se fane et des petites graines sont emportées par le vent. Le discours généralisant ramène à une perspective plus documentaire, mais la visée esthétique demeure comme le montrent par exemple dans le texte la métaphore du «ballet tourbillonnant des feuilles » et dans l'illustration l'image de la fleur plus haute que les arbres.

\section{Un album « mixte »}

S'il n'y a pas de monde fictionnel sans monde réel [5], la fiction et le réel s'articulent de manière particulière dans ce type d'album « composite », défini à la fois par le pluricodage et la polyphonie [6]. Cette polyphonie est illustrée ici par une double visée, fictionnelle et informative.

Nous qualifierons donc d'album «mixte » tout album qui manifeste cette double visée entrainant des choix structurels et discursifs particuliers.

Ces choix prennent ici la forme de deux bivalences, celle de la structure et celle de l'énonciation.

\subsection{Une visée double}

La visée double se vérifie dans cet album, où il s'agit d'une certaine manière d'instruire et de plaire, ce qui se traduit notamment par une pseudo-oralité, à travers les commentaires du narrateur (« bientôt il sera trop tard !»), qui semblent s'adresser directement au lecteur.

Dans la mesure où, avant dix ans, les enfants semblent « davantage concernés par la dimension narrative de l'album même si la curiosité scientifique est par moments bien réelle » [7], la fiction apparaît comme le passage obligé de la croyance au savoir.

Elle est pourvoyeuse de mondes possibles auxquels l'enfant peut adhérer dans une première lecture naïve, qu'il s'agira ensuite de confronter à la réalité pour passer de la croyance au savoir [8].

\subsection{Une structure double}

Comme pour l'album Un poisson est un poisson, de Leo Lionni, ainsi que l'ont observé Bruguière et alii [8], le récit permet ici la superposition d'un schéma narratif propre au conte et d'un phénomène biologique cyclique qui tous deux passent par une transformation qui s'inscrit dans un déroulement chronologique.

Dans l'album de Leo Lionni, les deux individus sur lesquels focalise la narration sont représentatifs de l'espèce. Dans Une si petite graine, d'Eric Carle, au contraire, cohabitent le générique et le singulier. L'album combine ainsi des mondes alternatifs, l' « alternativité » (pour reprendre l'une des propriétés 
caractérisant selon Hintikka, $1989^{4}$, la sémantique des mondes possibles) se présentant comme « une règle de construction d'un monde possible sur la base d'un autre monde » [8], en l'occurrence ici le monde réel. Cette même « alternativité » permet de pousser plus avant la fictionnalisation au moment de la transformation de la plante en fleur qui doit autant au phénomène biologique qu'au merveilleux des contes.

C'est cette coexistence de plusieurs mondes dans lesquels se déroule la croissance aboutie de la plante, contaminée par le merveilleux, qui est ici constitutive de la fiction.

\subsection{Une énonciation double}

Dès la première page, on remarque que cet album comprend à la fois des éléments de discours (le temps de base utilisé est le présent et l'énonciateur manifeste son attitude vis-à-vis du destinataire par le biais de phrases exclamatives et interrogatives) et des éléments de récit (emploi de la troisième personne, emploi de marques spatio-temporelles).

Adam et alii [9] ont proposé une analyse qui nuance le système discours/récit telle que l'a élaborée Benveniste [10]. Ils distinguent quatre sous-systèmes qui prennent en compte à la fois le degré d'implication de l'énonciateur dans son énoncé et le degré de distanciation par rapport au monde actuel du même énonciateur. A côté du « discours pur», les auteurs font ainsi figurer le « discours théorique », étroitement lié au monde actuel comme le discours pur, mais avec un énonciateur non impliqué. De la même manière, ils placent à côté du « récit pur », la «narration liée », éloignée du monde actuel comme le récit pur, mais avec un énonciateur impliqué.

Or cet album nous paraît relever d'un cinquième sous-système, assez fréquemment représenté en littérature de jeunesse dans ce type d'albums, et qui mixerait deux des sous-systèmes identifiés par ces linguistes.

Observons par exemple l'incipit :

«C'est l'automne.

Le vent souffle. Il soulève les graines de fleurs

au dessus du sol et les emporte. Parmi elles,

il y en a une qui est toute petite, minuscule !

Est-ce qu'elle parviendra à suivre les autres?

Et d'ailleurs où vont-elles, toutes ces graines?»

L'énonciation double entremêle ici le temps du « discours théorique » (le présent gnomique) porté par un énonciateur « informateur », avec les temps de la «narration liée » (passé composé/présent narratif/futur) porté par un énonciateur narrateur qui reste cependant sur une énonciation distanciée. De cette double valeur du présent émane la structure mixte de l'album qui suit à la fois un schéma narratif rapportant les aventures du personnage « une toute petite graine » et le déroulement cyclique de la vie des plantes.

\subsection{Un support « composite » propice à la co-construction des inégalités scolaires}

Cet album présente donc des caractéristiques que nous avons ailleurs identifiées comme celles du support « composite » en littérature de jeunesse [6] : hétérogénéité liée au pluricodage et hétérogénéité discursive, que traduisent les spécificités linguistiques définies ci-dessus.

\footnotetext{
${ }^{4}$ J. Hintikka. L'intentionnalité et les mondes possibles. Lille : Presses universitaires de Lille (1989).
} 
Des recherches en cours [11, 12] montrent que ce type de support, inhérent à la littératie scolaire comprise comme l'ensemble des exigences de lecture requises à l'école aujourd'hui [13-15], nécessaire dans la perspective d'une maitrise par les élèves d'une complexité renouvelée, semble potentiellement propice à participer à la co-construction des inégalités scolaires, conjugaison de facteurs socio-culturels et de facteurs proprement scolaires [16-18].

\section{L'album dans la classe}

\subsection{Corpus}

Notre recherche, relevant de l'appropriation de l'écrit au cycle 2, concerne une classe de CP (19 élèves) située en ZEP, à Strasbourg. Elle porte ici sur une séquence réalisée en juin $2012^{5}$ et visant à la fois la découverte du monde et l'apprentissage de la lecture, en s'appuyant sur l'album Une si petite graine.

Cette séquence est composée de quatre séances, qui ont été enregistrées. À trois de ces séances sont associées des fiches de synthèse remplies par l'élève. Ces fiches arrivent en fin de séance après des échanges oraux collectifs et fixent les éléments à retenir.

Nous disposons donc de ces fiches ainsi que de la transcription des séances et des rappels de récit individuels effectués par le chercheur, enregistrés et transcrits.

\subsection{Qu'en fait l'enseignant ?}

Compte tenu du fait qu'en cycle 2, l'enseignement des sciences relève d'un domaine d'apprentissage transversal, non constitué encore en discipline (il prend la forme de « Découvrir le monde du vivant, de la matière et des objets » dans le domaine « Découverte du monde »), ce type d'album semble particulièrement bien convenir : le travail de l'enseignant montre qu'il utilise cet album à la fois pour faire lire les élèves, mais aussi pour enseigner la connaissance du monde. Son objectif est donc double.

C'est pourquoi nous nous interrogerons sur ce qu'il fait de la fiction dans cette perspective. Pour cela, nous examinerons conjointement le déroulement des séances et le contenu des fiches de synthèse.

La première séance se déroule selon une démarche habituelle dans les classes lorsqu'il s'agit d'albums de fiction, démarche dont rend bien compte la fiche $\mathrm{n}^{\circ} 1$ :

- lecture-compréhension thématique à partir de l'observation de l'illustration de la page 2 afin de construire un horizon d'attente ( « Que vois-tu sur cette illustration ? De quoi va parler le livre ?») ; cependant le lien n'est pas fait avec le titre au cours de la séance, ce qui permettrait de comprendre de quoi il est question dans l'album.

- observation du livre comme objet : cette approche, inspirée des travaux de Genette sur le paratexte, est utilisée par l'enseignant pour faire acquérir aux élèves un vocabulaire à ses yeux essentiel : auteur, illustrateur, maison d'édition, ISBN.

- travail sur l'encodage : relevé des graphies d'un son dans un extrait de l'album.

La séance 2 est consacrée dans un premier temps à une relecture magistrale d'une partie du texte déjà lue par les élèves, suivie d'un oral collectif.

La transcription montre un nombre élevé de digressions de la part de l'enseignant, qui peut s'expliquer par au moins trois raisons :

- la volonté de laisser les élèves s'exprimer quoi qu'ils disent (il acquiesce à tout ce qu'ils disent, même si c'est faux);

- la volonté de faire appel à leurs connaissances pour qu'ils se sentent impliqués ;

\footnotetext{
${ }^{5}$ Nous remercions Caroline Viriot-Goeldel, Université Paris Est, EA 4384 Circeft, Université Paris 8, UPEC, qui a recueilli et constitué ce corpus.
} 


\section{SHS Web of Conferences}

- l'absence d'objectif précis concernant la compréhension de l'album que manifestent les discussions au gré des interventions parfois hors de propos des élèves.

A la fin de ce premier temps, l'enseignant récapitule le début de l'histoire en individualisant le sort réservé à chacune des graines, distinguant trois destins qui avortent et mettant en valeur le quatrième, celui de la petite graine. Pour cela, il utilise systématiquement le mot « histoire».

M-C : Donc la première graine qu'est-ce qui lui est arrivé ?

E-C : Elle s'est brûlé au soleil.

M-C : Elle s'est brûlé au soleil et la deuxième est tombée sur une montagne. On continue. Et là, très bien Sarah. Là y en a une qu'est-ce qui lui arrive ? Elle tombe dans l'eau.

$\mathrm{M}-\mathrm{C}:$ Et la petite, qu'est-ce qui se passe avec la petite ? Elle est toute en bas là oui.

E-C : Mais c'est pas dangereux.

M-C : Mais elle ne tombe pas dans l'eau, la petite elle est toujours là. On nous dit juste : emportées par le vent, les autres continuent mais la plus petite ne vole pas aussi haut qu'elle. Pour la plus petite c'est un petit peu plus difficile. Mais elle est quand même toujours là. Alors que là on a quand même déjà trois graines en moins. (...)

M-C : C'est tout le début de l'histoire, ce qu'on vient de lire jusqu'à aujourd'hui, jusqu'à ce matin. Et il s'agit donc de compléter, avec les bons mots de l'histoire.

La fiche 2, introduite à l'issue de ce premier temps, propose un texte à trous, un extrait de l'album dans lequel les élèves doivent remettre individuellement des mots clés.

Le deuxième temps de la séance est construit autour de questions de compréhension portant sur l'aspect informatif de l'album. Après des échanges collectifs, les réponses aux questions sont écrites au tableau, et chaque élève doit ensuite les copier sur la fiche 2.

Si la première séance envisageait l'album du point de vue narratif, la seconde insiste surtout sur son aspect informatif : l'enseignant rend ainsi compte du double aspect d'Une si petite graine, mais le traite de façon implicite, sans que la correspondance des deux types de cycles ne soit explicitée.

M-C : Puis le printemps arrive. Et là qu'est-ce qui se passe avec les petites graines ? Antonella, qu'est-ce qui se passe avec les graines au printemps?

Antonella-C : Elles poussent.

M-C : Elles poussent oui. Est-ce que nous on avait pas planté dans le jardin certains choses avant l'hiver ? Et après il s'est passé quoi ?

E-C : Elles ont poussé un peu.

M-C : Ouais pendant tout l'hiver y a rien eu. Vous vous rappelez vous avez planté au début de l'année. (...)

M-C : On les avait plantés en automne. Pendant tout l'hiver il n'y a rien eu et quand y a eu le printemps les jonquilles sont sorties. (...)

M-C : Le printemps arrive, les graines poussent. Est-ce qu'on appelle ça encore des graines ?

EE-C : Non.

M-C : Ca devient des plantes. Qu'est-ce qu'il se passe ? Elles deviennent plus grosses et après ?

$\mathrm{M}-\mathrm{C}:$ Kenza.

Kenza-C : Après ça fait des fleurs.

M-C : Elles se transforment en fleurs et il va quand même y avoir un souci, vous avez remarqué qu'à chaque page il y a un autre souci pour ces pauvres graines.

Les deux séances suivantes sont consacrées à l'avancée de la découverte de l'album par la lecture à voix haute des élèves en groupes de niveaux et la relecture magistrale. Ces deux séances mettent en évidence le double objectif ici poursuivi : l'apprentissage de la lecture comme décodage et la construction de connaissances en « découverte du monde ». La dernière fiche illustre particulièrement ce dernier point : elle propose en effet un travail de production d'écrit à partir de quatre illustrations de l'album associées à quatre phrases écrites par l'enseignant et indiquant chacune des saisons. L'élève doit rendre compte de ce qu'il a retenu concernant le cycle de la croissance.

Les transcriptions de séances et les fiches recueillies montrent que l'objectif premier de l'enseignant est l'apprentissage de la lecture : séances de lecture à voix haute pour les élèves qui visent principalement 
le décodage, séances d'écriture consistant soit à copier, soit à encoder ou encore à faire des phrases. Cela permet de vérifier de nouveau que si la classe de CP est bien conçue par les enseignants comme la classe où l'on apprend à lire, cet apprentissage se restreint très souvent aux processus de bas niveau, englobant une conception de l'écriture fondée sur le produit bien plus que sur le processus.

Toutefois on ne peut pas dire que l'objectif de compréhension ne soit pas présent : il l'est principalement à l'oral et vise la connaissance du monde. En effet, parallèlement à l'apprentissage de la lecture, on perçoit un souci épistémologique chez l'enseignant : apprendre aux élèves la croissance d'une plante, mais en laissant de côté la visée fictionnelle de l'album, pourtant au départ présenté comme une histoire.

\subsection{Qu'en font les élèves ?}

\subsubsection{Protocole}

Partant du travail de l'enseignant qui focalise l'attention des élèves sur le rôle des saisons et le caractère cyclique de la croissance des plantes, il a paru pertinent au chercheur de mesurer leur niveau de compréhension du réel à partir de cet album.

Tous les élèves ont donc été interrogés individuellement par le chercheur. Il a sollicité un rappel du récit («Est-ce que tu pourrais me raconter cette histoire ?»), en prévoyant des relances ponctuelles portant plus spécifiquement sur le phénomène biologique («Qu'est-ce que devient la petite graine ?»). Sur les dix-neuf rappels recueillis, nous en avons sélectionné quatre, représentatifs des résultats obtenus dans la classe, d'une compréhension maximale à une compréhension minimale.

\subsubsection{Cadre d'analyse}

Nous considérons que la clé de la compréhension de l'album est la collusion entre la transformation propre au récit (qui fait du personnage un héros) et la transformation propre au phénomène biologique de la croissance (ici la graine devenue plante et fleur) : c'est en cela que la structure narrative peut être facilitatrice, si elle est traitée comme telle.

Nous avons donc retenu deux types de critères d'analyse.

Le premier porte sur la connaissance du monde.

Le phénomène de la croissance s'appuie sur le principe de transformation et le principe cyclique (recommencement lié aux saisons) que nous retiendrons pour évaluer le degré de connaissance du monde des élèves à l'issue de la lecture de l'album.

Le second relève du rôle de la fiction, en tant qu'accès au savoir.

Nous retiendrons comme critères principaux d'une part la singularité liée à la petite graine en tant que personnage (croissance présentée comme un destin particulier) et d'autre part son statut d'héroïne de conte (reconnaissance admirative de tous à l'issue de sa croissance qui relève d'une apothéose merveilleuse : «bientôt jusqu'au ciel »).

\subsubsection{Résultats}

L'étude des rappels nous a permis de déterminer deux pôles : d'un côté les élèves qui se servent de la structure mixte de l'album et qui approchent le plus finement la notion de cycle ; de l'autre côté, ceux qui ne prennent pas du tout en compte la fiction et dont le rappel semble s'appuyer sur des connaissances antérieures, notamment en ce qui concerne la transformation de la graine en plante. 
Nous avons ainsi identifié quatre profils d'élèves, représentés ici par Ibrahim, Alvyn, Leila et Assia.

\begin{tabular}{|l|l|l|l|l|}
\hline Elèves & $\begin{array}{l}\text { Connaissance du } \\
\text { monde } \\
\text { principe de } \\
\text { transformation }\end{array}$ & $\begin{array}{l}\text { Connaissance du } \\
\text { monde } \\
\text { principe cyclique }\end{array}$ & $\begin{array}{l}\text { Fiction } \\
\text { petite graine } \\
\text { personnage }\end{array}$ & $\begin{array}{l}\text { Fiction } \\
\text { petite graine } \\
\text { héroïne } \\
\text { Merveilleux } \\
\text { /Admiration }\end{array}$ \\
\hline Ibrahim & oui & non & non & non \\
\hline Alvyn & oui & non & non & non \\
\hline Leila & oui & oui & oui & non \\
\hline Assia & oui & oui & oui & non (M) / oui (A) \\
\hline
\end{tabular}

On observe quatre types de réponse chez ces élèves :

- un récit souvent incomplet et fragmentaire, où les élèves essaient de se souvenir du contenu de l'album ; les relances, nombreuses, font simplement apparaître que le phénomène de la transformation est déjà connu et que l'album n'apporte pas de connaissances nouvelles, comme chez Ibrahim (sept élèves sont dans ce cas) :

«Ça parle d'une petite graine. La petite graine, elle peut pas voler si haut. Et une graine se fait brûler par le soleil. Une autre graine tombe sur l'eau. Une autre qui s'écrase sur la montagne. L'autre elle s'écrase dans l'eau.

Et notre toute petite graine, qu'est-ce qu'elle devient?

Une fleur. »

- un récit plus complet où seul le point de vue biologique est envisagé, comme chez Alvyn, qui privilégie la généralité au détriment du statut fictionnel de la petite graine (un seul élève est dans ce cas) :

«Il y a une plante qui pousse trop vite et il y a une plante qui peut pas pousser parce qu'il a pas de l'eau ni du chaud... du soleil. Alors, il va mourir, et les autres y va pas mourir parce qu'il a de l'eau et du soleil. Après il y a la grande plante il est encore plus grand plus grand plus grand et bientôt jusqu'au ciel et les autres ils sont plus petits. Et c'est tout. »

La fin de l'entretien semble montrer qu'Alvyn n'a pas compris la notion de cycle : la fiction incite en effet au marquage temporel, ce que traduit la prise en compte des saisons et notamment le retour à l'automne dans d'autres rappels.

- un récit en deux temps, qui privilégie encore l'aspect biologique. Passant par l'individualisation, il s'attache à ce qui se passe pour chacune des graines, avant de se focaliser sur le sort de la petite graine. Le rappel de Leila en est un bon exemple :

«Et il en reste une dernière, elle devient immense, elle est née de la toute petite graine. »

Ce profil est majoritairement représenté dans le groupe (sept élèves sont dans ce cas).

- un récit où l'on observe le même va et vient du général au singulier que dans l'album, comme chez Assia qui fait alterner connaissance du monde et fiction, ce que montrent et l'emploi contrasté du déterminant (indéfini vs. défini) et la restriction («mais c'est pas ») :

«Et après il y a l'oiseau il picore une graine, mais c'est pas la toute petite graine, parce qu'il la voit pas encore. » (quatre élèves sont dans ce cas).

Les élèves qui ont le mieux compris le phénomène biologique sont ceux qui sont passés par la fiction, ce qui suppose qu'ils ont su articuler d'eux-mêmes la double causalité de l'évolution (croissance/saisons). Or il s'agit aussi des élèves placés dans le groupe « des plus forts » (les « verts ») dans cette classe où l'enseignant a réparti ses élèves en groupes de niveau. A l'inverse, Ibrahim et Alvin appartiennent aux deux groupes les plus en difficulté (les « rouges »). 
La fiction nous semble avoir facilité le passage de la croyance à la connaissance pour les élèves qui ont pu tirer le meilleur parti de ce support, en leur permettant d'aller du particulier au général et en favorisant le marquage temporel. Ce n'est pas le cas des élèves les plus faibles. Cela nous paraît confirmer l'effet différenciateur d'un tel support, tel qu'il est ici travaillé.

Cependant notre étude est qualitative, et il n'y a eu ni pré-test ni post-test qui permettent de confirmer les acquisitions scientifiques des élèves. En outre, il est vraisemblable que tous les élèves aient été au fait du principe de transformation propre à la croissance, largement enseignée dès la maternelle. De même, si certains élèves ont bien compris le principe d'un recommencement, il n'est pas possible de savoir si ce recommencement, inhérent à la notion de cycle, est perçu comme celui de l'espèce et non celui de l'individu.

\section{Conclusion}

L'approche didactique n'a pas pris en compte la nature spécifique de cet album «mixte », dont le composite repose sur une visée double, un schéma double et une énonciation double. L'enseignant a opéré un choix de lecture en lien avec les programmes : il n'a traité l'aspect fictionnel de l'album qu'incidemment, et a centré sa mise en œuvre sur la construction de connaissances du monde du vivant.

Mais les rappels des élèves montrent que ceux qui ont identifié cet aspect fictionnel perçoivent la croissance de la manière la plus précise, même si l'album présente des aspects contestables du point de vue scientifique. Nous avons ainsi pu constater que comprendre la structure narrative permet de comprendre la structure cyclique, en particulier grâce à l'ancrage temporel.

Afin que tous les élèves construisent des connaissances avec ce type d'album, autrement différenciateur, et en nous appuyant sur les résultats ci-dessus, la spécificité « composite » d'un album de type « mixte » doit être prise en compte par le médiateur qu'est l'enseignant à l'école. Nous supposons par exemple qu'il serait pertinent de traiter un tel album comme un album de fiction, qui ouvre à une expérimentation des possibles, en focalisant d'abord sur l'histoire de la petite graine, pour ensuite montrer que si en tant que personnage elle n'existe pas dans notre monde (on ne connait pas de plante qui grimpe jusqu'au ciel), en revanche elle présente des caractéristiques qui relèvent bien du monde du vivant, que les élèves connaissent.

\section{Références}

[1] É. Bautier, J. Crinon, C. Delarue-Breton, et B. Marin. Les textes composites : des exigences de travail peu enseignées ? Repères, 45, 63-80 (2012)

[2] D. Cohn. Le Propre de la fiction. Paris : Seuil (2001)

[3] É. Bautier, D. Manesse, B. Peterfalvi et A.Verin. Le cycle du cerisier : une narration scientifique ? Repères, 21, 146-164 (2000)

[4] C. Sauzeau et É. Triquet. L'atelier d'écriture d'un récit de fiction scientifique : un dispositif interdisciplinaire pour un nouveau genre ? Actes du 9e colloque de l'AIRDF, Québec, 26-28 août 2004 (2004)

[5] U. Eco. Lector in fabula. Paris : Grasset (1985)

[6] P. Richard-Principalli et M.-F. Fradet (2012, à paraître). Complexité littéraire et littératie au début de l'école élémentaire. Symposium « Supports didactiques, pratiques d'enseignement et préoccupations sociologiques sur les inégalités d'apprentissage », $\mathrm{XVII}^{\mathrm{e}}$ congrès international de l'AMSE (Association mondiale des sciences de l'éducation), 3-8 juin 2012

[7] N. Auger et D. Jacobi. Autour du livre scientifique documentaire : un dispositif de médiation entre adulte et enfant lecteur. Aster, 37, 219 (2003)

[8] C. Bruguière, J.-L. Héraud, J.-P. Errera et X. Rembotte. Mondes possibles et compréhension du réel : La lecture d'un album en cycle 2 comme source de questionnement scientifique. Aster, 44 , 72-73 (2007) 


\section{SHS Web of Conferences}

[9] J.-M. Adam, G. Lugrin et F. Revaz. Pour en finir avec le couple récit / discours. Pratiques, 100, 81-98 (1998)

[10] É. Benveniste. Problèmes de linguistique générale. Paris : Gallimard (1966)

[11] C. Delarue-Breton et É. Bautier. Restitution de récit et nouvelle littératie scolaire. Étude comparée de productions orales d'élèves issus d'établissements scolaires de milieux contrastés. 9th IAIMTE Internatioanl Conference (International Association for the Improvement of Mother Tongue Education), 11-14 juin

[12] C. Viriot-Goeldel C. et C. Delarue-Breton. Des textes composites à l'école : nouvelle littératie scolaire, apprentissages et inégalités. Spirale, 53, 21-31 (2014)

[13] É. Bautier. Pédagogie invisible et littératie étendue. Construire des significations scolaires à partir de supports composites. Communication au congrès de l'Association Française de Sociologie, Nantes, 2-5 septembre (2013)

[14] É. Bautier et C. Delarue-Breton. Littératie scolaire et textes composites au cycle 2 de l'école primaire : l'exemple de la lecture d'un manuel scolaire de SVT. Symposium " Pour une construction empirique de l'archi-élève lecteur et scripteur ». REF Genève, 9-10 septembre (2013)

[15] J. Crinon et P. Richard-Principalli. Littératie scolaire et textes composites au cycle 2 de l'école primaire : l'exemple d'albums de jeunesse. Symposium «Pour une construction empirique de l'archi-élève lecteur et scripteur ». REF Genève, 9-10 septembre (2013)

[16] J.-Y. Rochex et J. Crinon (Dir.). La construction des inégalités scolaires. Au cœur des pratiques et des dispositifs d'enseignement. Rennes : Presses Universitaires de Rennes (2011)

[17] É. Bautier et J.-Y. Rochex. Activités conjointes ne signifie pas significations partagées. Raison éducative, 8, 199-220 (2004)

[18] É. Bautier et R. Goigoux. Difficultés d'apprentissage, processus de secondarisation et pratiques enseignantes : une hypothèse relationnelle. Revue Française de Pédagogie, 148, 89-100 (2004) 